\title{
Performance Evaluation of Nested Watermarked Scheme using Objective Image Quality Metrics
}

\author{
Priya R Sankpal \\ Department of Electronics and Communication Engineering \\ BNM Institute of Technology, Bengaluru, Karnataka, India \\ Email-priyarsankpal@bnmit.in \\ P. A. Vijaya \\ Head of Department, Department of Electronics and \\ Communication Engineering, BNM Institute of Technology, \\ Bengaluru, Karnataka, India \\ Email-pavijaya@bnmit.in
}

\begin{abstract}
In this digital era, extensive usage of internet has resulted in images being subjected to various distortions during processing and transmitting over open networks. For watermarking applications, watermarked image quality is evaluated in comparison to the original image. In the last decade literature, many performance metrics have been used, comprising of both subjective and objective quality metrics. The subjective image quality metrics are generally time consuming and expensive in contrast to objective image quality metrics. This paper, attempted an investigation for measuring performance of nested watermarking schemes and objective image quality metrics which included Peak signal to Noise Ratio (PNSR), Mean Square Error (MSE), Correlation Coefficient $(C C)$, Normalized Correlation Coefficient (NCC) and Structural Similarity Index (SSIM). For the proposed nested watermarking method, these objective image quality metrics were evaluated using combination of Discrete Wavelet Transforms (DWT) and Singular values (SVD) in the four frequency bands. Performance of objective zmetrics can be judged based on the their sensitiveness to watermarking artifacts. It is clear from the results obtained, for most watermarking applications SSIM provides better insight into the performance of the algorithm used where as PNSR fails for certain watermark artifacts. Findings drawn in this paper are based on the experimentation of nested watermarking algorithm using a DICOM image database.
\end{abstract}

\section{Keywords: Nested Watermarking, Objective image quality metrics, DWT, SVD, PNSR, SSIM}

\section{INTRODUCTION}

In the digital era, easy access to internet and image processing, privacy and security of data are major concern. In maintaining the upcoming threats to digital information, Digital image watermarking provide aide in tamper resistance, ownership, content authentication and protection of information. When implementing a watermarking scheme, different requirements need to be considered based on the applications involved. The essential requirements of watermarking schemes are imperceptibility, payload and robustness. These requirements play a pivotal function in evaluating watermarking system performance. Often these requirements are conflicting and trade-offs between them are considered based on the watermarking system applications. A watermarking scheme is said to be effective when there is a balance between these requirements. Research literature in this area has discussion of numerous transformation techniques that satisfy requirements of watermarking system. One of the most preferred combination for watermarking process is the Discrete Wavelet Transforms (DWT) and Singular Values Decomposition (SVD) [1]. 


\subsection{Discrete Wavelet Transforms}

Discrete wavelet transform (DWT), mathematically is wavelet transform which decomposes the signal into wavelets, instead of frequencies. Wavelets in DWT are sampled discretely. DWT fairs better over other Fourier transforms like Discrete Cosine Transform (DCT) and Discrete Fourier Transform (DFT), in terms of temporal resolution. DWT captures multiple information such as time and frequency location. DWT based image watermarking techniques decompose the image into different frequency sub band levels. The sub bands frequencies cover the wide range of the spectrum of the image [1]

\subsection{Singular Value Decomposition}

Singular values are the useful tools of linear algebra that finds application in various signal processing operations including image watermarking. Singular values of any image (of the dimension i.e. $\mathrm{mxm}$ ), is given as:

Image $=\mathrm{U} * \mathrm{~S} * \mathrm{~V}^{\mathrm{T}}$

where $\mathrm{U}$ and $\mathrm{V}$ are the orthogonal elements and $\mathrm{S}$ are the diagonal elements. Choice of SVD for image watermarking applications is mainly because, they represent a large portion of the signal energy, are fairly immune to noise, i.e. singular values of the image do not change significantly when a small perturbation is added to image intensity and singular values can be used with square and rectangular images. SVD based watermarking techniques robustness is found to be fairly good when used in combination with transform domain techniques [2].

In this paper, methodology section describes the proposed DWT and SVD combination based nested watermarking scheme. In the results section, image quality assessment of the proposed scheme is validated using objective image quality metrics: Peak-Signal-to-Noise Ratio (PSNR), Mean Squared Error (MSE), Structural Similarity Index (SSIM), and Normalized Cross-Correlation (NCC). Proposed scheme is also tested for robustness as well as perceptual transparency by subjecting the watermarked image to different attacks. Lastly in the conclusion section, image quality metrics are compared with regard to the application of the watermarking system.

\section{Methodology}

In the proposed method, an attempt was made for studying the performance of multiple watermarks that were embedded in nested pattern. Research work carried in this field suggests different transformation techniques available for image watermarking. Recent developments in this field show that, combination of Discrete Wavelet Transform (DWT) and Singular Value Decomposition (SVD) have been the most preferred one. [3]

For implementing the multiple watermarks in the nested pattern Discrete Haar Wavelet Transforms was used in combination with Singular Value Decomposition (SVD). Watermark embedding in nested pattern was performed as discussed below. Inputs to the nested watermarking algorithms are the cover image (CI), watermark image 1 (WM1) and watermark image 2 (WM2). Image data base used for testing the performance of nested scheme comprised of 10 different DICOM images with dimension of 256x256 in Portable Network Graphic (PNG) format. Both the cover image and watermark images were chosen of the same size. Figure 1 shows the DICOM images used as cover image and the two different watermark images. 


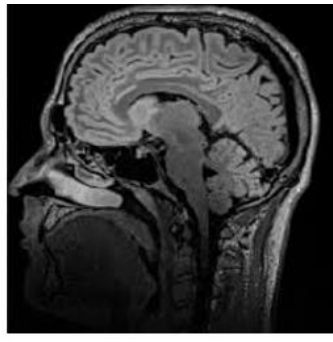

Cover Image for Watermarking

(A)

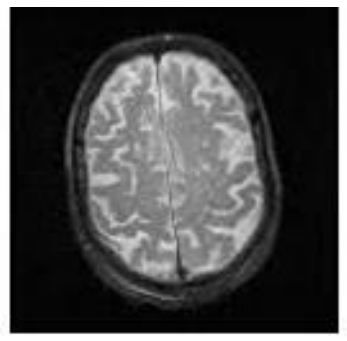

Watermark Image 1

(B)

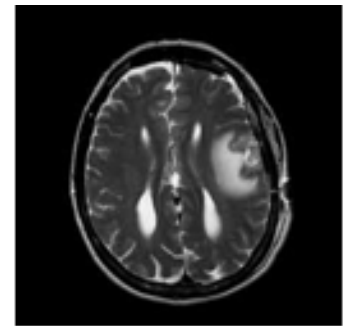

Watermark Image 2

(C)

Figure 1. DICOM Images used as input for Nested watermark algorithm

\subsection{Nested Watermark Scheme 1}

In this method, watermark image 1 is embedded or watermarked into cover image by applying Haar DWT to the cover image 2. Cover image is decomposed into four bands of different frequencies as: LL,HL,LH and HH. The different frequency bands are termed as low frequency band (LL) which provides approximate details of the image, mid frequency bands ( $\mathrm{HL}$ and $\mathrm{LH}$ ) give the horizontal and vertical details of the image and high frequency band $(\mathrm{HH})$ which provide the diagonal details of the image. In the proposed method, Singular values of cover and watermark images are computed for all the four bands. Using the singular values of the watermark image, cover image singular values are modified during the embedding process. In this method, firstly an attempt was made to embed the watermark in the LL frequency band using SVD.

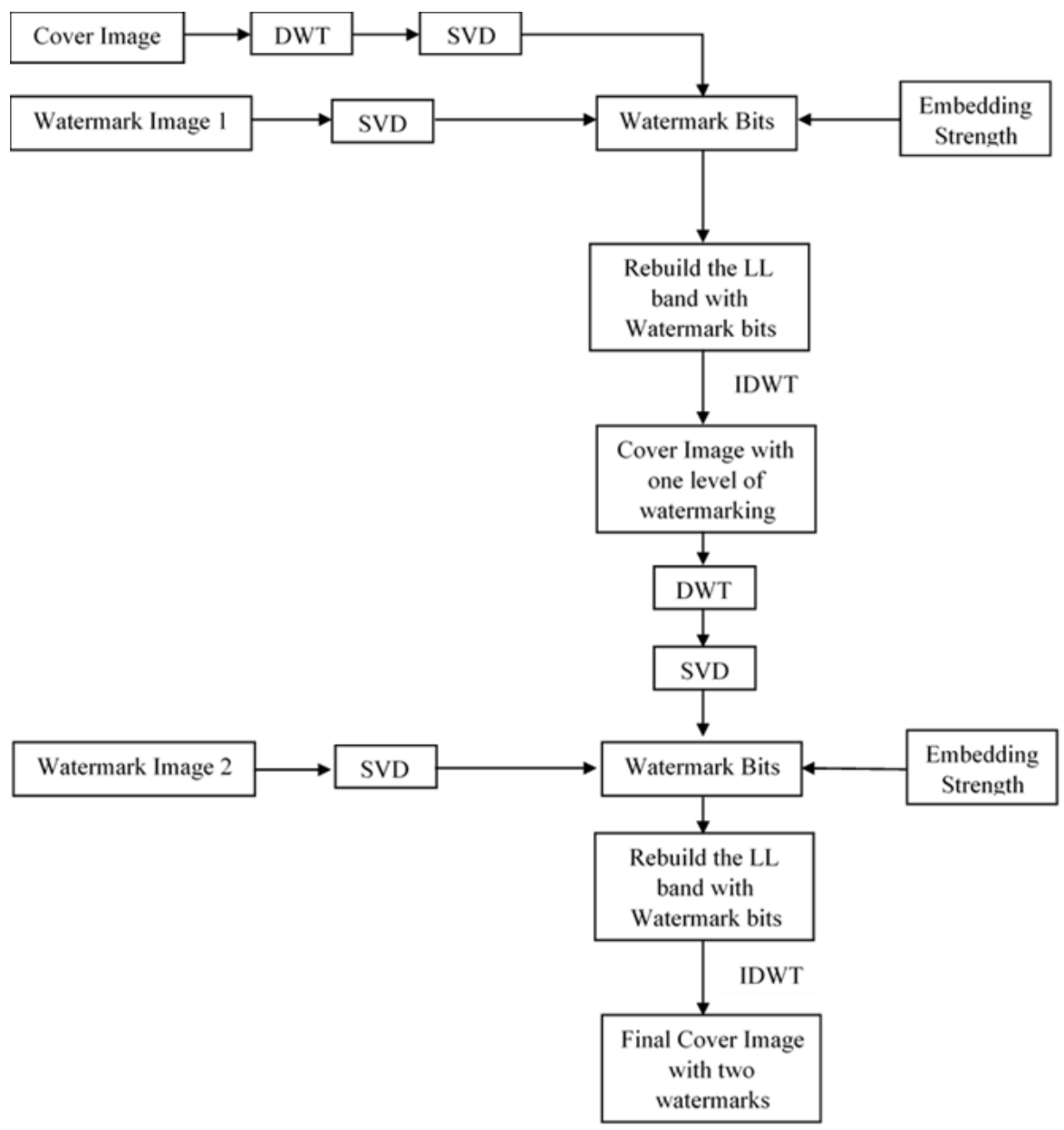

Figure 2. Overview of Proposed System 
Singular values of cover image and watermark image computed were used along with embedding strength in calculating the watermarking bits to be embedded into cover image. The cover image LL band singular values were rebuilt with the modified singular values after the addition of watermark bits. This resulted in first level watermarked image. Watermark image 2 is embedded in the already watermarked cover image by again applying DWT and superseding the cover image LL band singular values with that of the watermark image 2 . Figure 2 shows the overview of the proposed algorithm. When inverse DWT was applied, the final output of the this method results in nested watermarking, with the cover image having two watermarks embedded in it. Watermarks (Watermark image 1 and Watermark image 2) used are pre-processed to have singular values that match with those of the target images (cover image and its modified versions). For the extraction of the watermarks from the cover image, again the combination of DWT and SVD is used, as discussed in the algorithms below:

\subsection{Algorithm : Watermark Embedding}

1. Read the cover image (CI).

2. Apply DWT to CI. Decomposition results in LL, HL,LH,HH bands.

3. SVD is applied to the LL band of the CI.

$$
\mathrm{LL}=\mathrm{Uc}^{*} \mathrm{Sc} * \mathrm{Vc}^{\mathrm{T}}
$$

4. Read the watermark image 1 (WM1) for first level of watermarking

5. Compute the SVD of watermark image 1(WM1)

$$
\mathrm{Wm} 1=\mathrm{Uw} 1 * \mathrm{Sw} 1 * \mathrm{Vw} 1{ }^{\mathrm{T}}
$$

6. Singular values of cover image LL band are superseded by the singular values of the WM1.

7. Compute watermarking bits for cover image as:

$$
\text { Smark1 = SVD (LL) + Embedding strength *SVD }(\mathrm{Wm} 1)
$$

8. Rebuild LL band of cover image by recomputing singular values as

$$
\text { LL_w1 }=\text { Uc* Smark } 1 * \mathrm{Vc}^{\mathrm{T}}
$$

9. Inverse DWT is applied to obtain the first watermarked image of cover image

10. Read the cover image again for $2^{\text {nd }}$ level of nested watermarking.

11. Apply DWT to cover image and decompose into four bands

12. SVD is applied to the LL band of the cover image.

$$
\mathrm{LLcw}=\mathrm{Ucw}^{*} \mathrm{Scw} * \mathrm{Vcw}{ }^{\mathrm{T}}
$$

13. Read the second watermark image 2 (WM2)

14. Compute the SVD of watermark image 2 (WM2)

$$
\mathrm{Wm} 2=\mathrm{Uw} 2 * \mathrm{Sw} 2 * \mathrm{Vw} 2{ }^{\mathrm{T}}
$$

15. Singular values of cover image LL band are superseded by the singular values of the WM2.

16. Compute watermarking bits for the final cover image as:

$$
\text { Smark2 = SVD (LLcw) + Embedding strength *SVD }(\mathrm{Wm} 2)
$$

17. Rebuild LL band of the final cover image by recomputing singular values as

$$
\mathrm{LL} \_\mathrm{w} 2=\mathrm{Uc} * \operatorname{Smark} 2 * \mathrm{Vc}^{\mathrm{T}}
$$

18. Inverse DWT is applied to obtain the final cover image with two nested watermarks

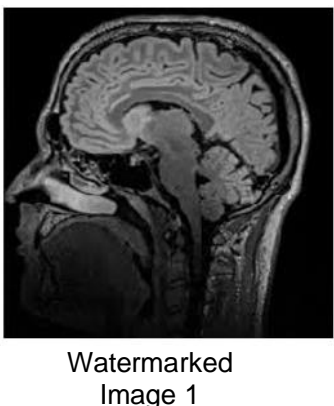

(A)

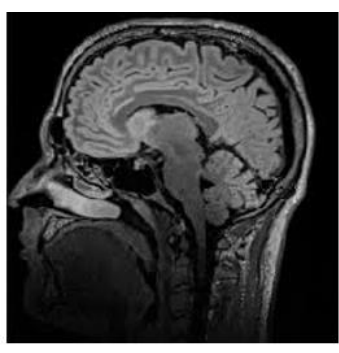

Final Cover Image with 2 nested watermarks

(B)

Figure 3. Watermarked Images at the intermediate stages of embedding algorithm implementation 
Figure 3 shows the watermarked images at two different levels of nesting the images. Image in Figure $3 \mathrm{~A}$ is obtained after the first watermark (Figure 1B) is embedded in the cover image (Figure 1A). Image in Figure 3B is obtained after the second watermark (Figure 1C) is embedded in the already watermarked cover image

\subsection{Algorithm : Watermark Extraction}

1. Read the cover image with two nested watermarks and apply inverse DWT to it

2. Compute Singular values of cover image (CI)

3. Compute watermark recovery bits, using singular values of cover image and WM2 as:

Swrec1 $=($ SVD $(\mathrm{CI})-$ SVD $(\mathrm{WM} 2)) /$ Embedding strength

4. Extract WM2 from the cover image, by computing singular values as:

$$
\mathrm{WM} 2=\mathrm{Uw} 2 * \mathrm{Swrec} 1 * \mathrm{Vw} 2^{\mathrm{T}}
$$

5. Read the extracted watermarked image WM2 and apply inverse DWT to it

6. Compute Singular values of watermarked image 2 (WM2)

7. Compute watermark recovery bits, using singular values of WM2 and WM1 as:

Swrec2 $=(\mathrm{SVD}(\mathrm{WM} 2)-\mathrm{SVD}(\mathrm{WM} 1)) /$ Embedding strength

8. Extract WM1 from the watermarked image 2 (WM2), by computing singular values as:

$$
\mathrm{WM} 1=\mathrm{Uw} 2 * \mathrm{Swrec} 1 * \mathrm{Vw}^{\mathrm{T}}
$$

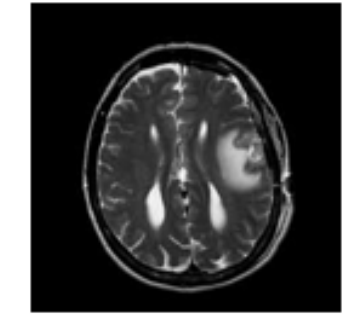

Extracted Watermark Image 2

(A)

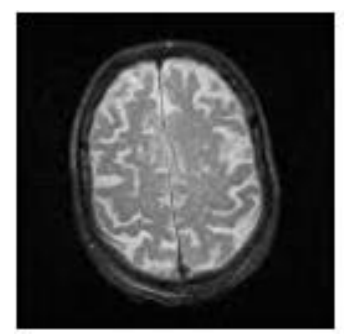

Extracted Watermark Image 1

(B)

Figure 4. Extracted Watermark Images at the intermediate stages of extraction algorithm implementation

Figure 4 shows the extracted watermark images at two different levels of nesting. Image in Figure $4 \mathrm{~A}$ is the extracted watermark image 2 obtained final cover image with 2 nested watermarks (Figure 3B) and the image in Figure 4B is obtained after extracting watermark image 1 from the image in Figure $3 \mathrm{C}$

\section{Experimental Results}

To measure the performance of watermarking algorithms, quality of the watermarked cover image is compared with the original watermark image in terms of image degradation or distortions. This is can be done with various quality assessment metrics which broadly fall under two categories: Subjective evaluation metrics and Objective evaluation metrics. Subjective evaluation involves measuring the statistical differences between the original image and the watermarked image in terms of visual impairments. There is no standard available for subjective evaluation process. In [4] authors consider the watermark process as a source of distortions and therefore evaluate the corresponding annoyance, by presenting images explicitly to the observer. Observers rate the impairments on a five scale as: imperceptible ( scale value 5), perceptible though not annoying ( scale value 4), slightly annoying (scale value 3 ), annoying (scale value 2 ) and very annoying (scale value 1 ). Watermarking algorithms with scale value higher than 4, perform fairly in terms of imperceptibility and robustness. However, Subjective evaluation process is expensive, tedious, time consuming and not suitable for real time based applications. Objective evaluation process assess the quality of the watermarking algorithms by using automatic tools such as: Peak Signal to Noise Ratio (PSNR), Mean Square Error 
(MSE), Normalized Correlation Efficient (NCC), Structural Similarity (SSIM), Universal Quality Index (UQI), Komparator, Reduced Reference Image Quality Assessment (RRIQA) and C4. In this section, the proposed nested watermarking scheme performance is evaluated using objective image quality metrics such as PSNR, NCC, SSIM [5].

\subsection{Peak Signal to Noise Ratio (PSNR) and Mean Square Error (MSE)}

Peak Signal to Noise Ratio (PSNR), Mean Square Error (MSE) are the widely used objective metrics and defined as

$$
\begin{aligned}
& \operatorname{MSE}(X, Y)=\frac{1}{N} \sum_{i=1}^{N} e_{i}^{2}=\frac{1}{N} \sum_{i=1}^{N}\left(x_{i}-y_{i}\right) 2 \\
& \operatorname{PSNR}(X, Y)=10 \log _{10} \frac{255^{2}}{\operatorname{MSE}(X, Y)}
\end{aligned}
$$

Where $\mathrm{X}$ is the original image and $\mathrm{Y}$ is the watermarked image. The error between $\mathrm{X}$ and $\mathrm{Y}$ is represented as ' $e$ '. ' $N$ ' represents number of pixels in the image [6].

\subsection{Structural Similarity Index (SSIM)}

The Structural similarity (SSIM) metric, measure the quality of original and watermarked image by capturing the similarity of images. Similarity is measured in terms of Luminance, contrast and structure. Luminance comparison function $l(\mathrm{X}, \mathrm{Y})$ for original image $\mathrm{X}$ and watermarked image $\mathrm{Y}$ is defined as

$$
l(X, Y)=\frac{2 \mu_{X} \mu_{Y}+C 1}{\mu_{x}^{2}+\mu_{y}^{2}+C 1}
$$

Where $\mu \mathrm{x}$ and $\mu \mathrm{y}$ are the mean values of $\mathrm{X}$ and $\mathrm{Y}$ respectively and $\mathrm{C} 1$ is the stabilization constant [7] in the watermark embedding process

Research work in the field of watermarking has suggested that the modification of LL band beyond certain limit results in degradation in perceptual quality of the image. However, with the method adopted, it was found that, embedding the watermark in LL band resulted in good imperceptibility of the final cover image in comparison to other band of frequencies. This is evident from the PNSR values obtained for all the different frequency bands as shown in Table 1.

\section{Table 1. PNSR values of different frequency bands for Nested Watermark Scheme}

\begin{tabular}{|c|c|c|}
\hline $\begin{array}{c}\text { Frequency } \\
\text { band }\end{array}$ & PSNR1 & PSNR2 \\
\hline LL & 53.6559 & 51.5558 \\
\hline HL & 55.5687 & 51.1725 \\
\hline LH & 55.7113 & 51.3690 \\
\hline HH & 55.6193 & 51.2734 \\
\hline
\end{tabular}

The nested watermark scheme performance was also evaluated against different values of embedding strength This evaluation test was carried for the decomposition level at LH band, as the imperceptibility of the image fairs better than the other band of frequencies. This evident from the PSNR values obtained for different bands as shown in Table 2. Table 2 shows that the PNSR value for embedding strength of 0.0001 shows a very good perceptual quality of the watermarked image. 
Table 2. Performance of Nested Watermark Scheme against Embedding strength for LH band

\begin{tabular}{|c|c|c|}
\hline $\begin{array}{c}\text { Embedding } \\
\text { strength }\end{array}$ & PSNR1 & PSNR2 \\
\hline $\mathbf{0 . 1}$ & 36.4198 & 32.2826 \\
\hline $\mathbf{0 . 0 1}$ & 55.7113 & 51.369 \\
\hline $\mathbf{0 . 0 0 1}$ & 77.7223 & 73.9911 \\
\hline
\end{tabular}

Correlation coefficient analysis was carried between the original and watermarked image for all the four different band of frequencies. In plain or the original images the correlation coefficients are close to 1 . With this implementation of nested watermarking scheme, very good imperceptibility is achieved, as the correlation coefficients and its normalized values (NCC) of the watermarked image are close to 1 as shown in Table 3. SSIM based on perception gives the similarity measures between the original and watermarked image. As shown in Table 3, the computed value of SSIM is close to 1 for all four band watermark embedding.

Table 3. Performance of Nested Watermark scheme for various objective image quality metrics

\begin{tabular}{|c|c|c|c|c|c|c|}
\hline $\begin{array}{c}\text { Frequency } \\
\text { band }\end{array}$ & CC1 & CC2 & NCC1 & NCC2 & SSIM & $\begin{array}{c}\text { Elapsed Time } \\
\text { in seconds }\end{array}$ \\
\hline LL & 1.000 & 1.000 & 1.000 & 1.000 & 0.9999 & 6.7309 \\
\hline HL & 1.000 & 0.999 & 1.000 & 0.999 & 0.9995 & 7.0519 \\
\hline LH & 0.999 & 0.999 & 0.999 & 0.999 & 0.9994 & 5.6342 \\
\hline HH & 1.000 & 0.999 & 0.999 & 0.999 & 0.9992 & 5.4067 \\
\hline
\end{tabular}

Robustness of the proposed nested watermark scheme was evaluated by observing the watermarking artifacts when subject to known set of attacks. Attacks such as noise addition, cropping, filtering, compression were applied. PNSR, NCC and SSIM are used as metrics to quantify the nested watermark scheme performance against the various attacks. Figure 5 shows the final watermarked image when subjected to different types of watermark artifacts

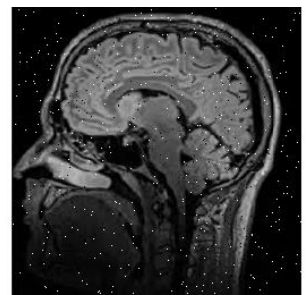

Salt \& Pepper: 0.01

(A)

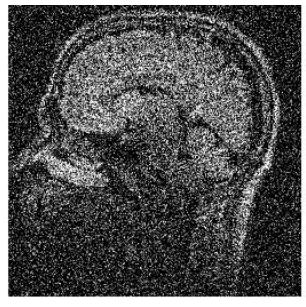

Gaussian Noise: 0.01

(D)

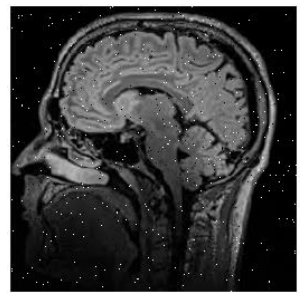

Salt \& Pepper: 0.1

(B)

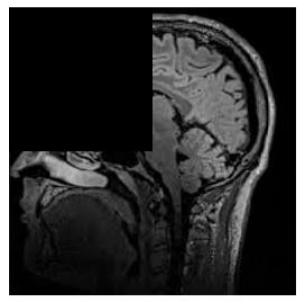

Cropping : $1: 128$

(E)

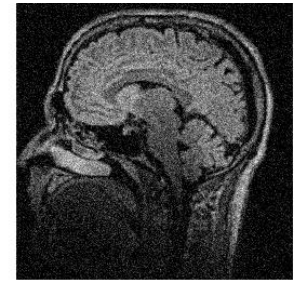

Gaussian Noise: 0.01

(C)

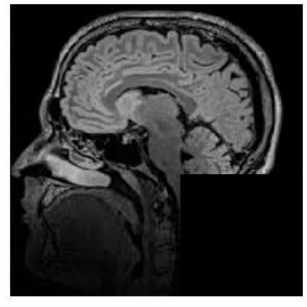

Cropping - 150:256

(F) 


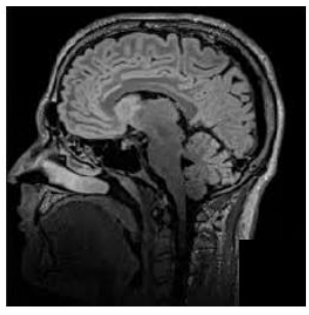

Cropping - 200:256

(G)

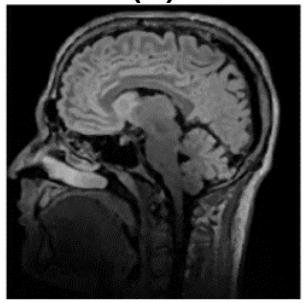

Median Filter

(J)

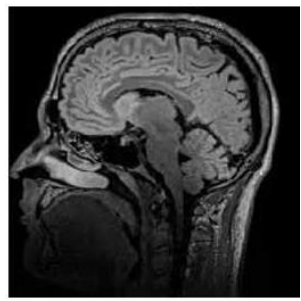

JPEG Compression with $Q=50 \%$

(M)

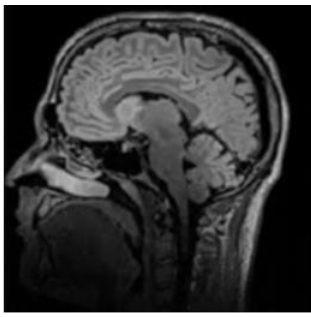

Blurring

(H)

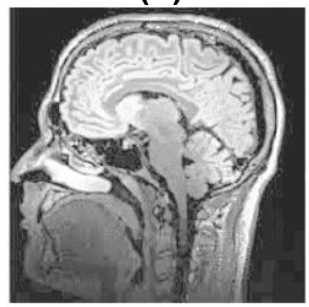

Histogram Equalisation

(K)

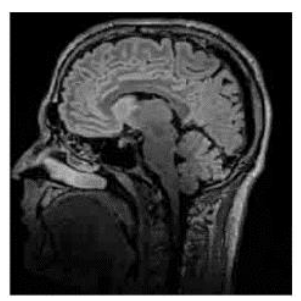

JPEG Compression with $Q=20 \%$

(N)

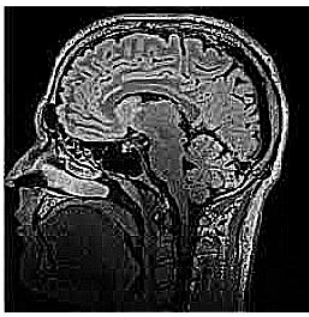

Sharpening

(I)

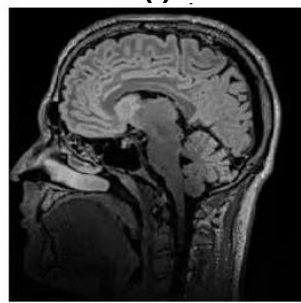

JPEG Compression with $\mathrm{Q}=80 \%$

(L)

Figure 5. Various watermark artifacts of the final watermarked cover image with 2 nested watermarks in it

The recorded values are as shown in table 4. Extracted watermarks after the various attacks are as shown in figure 4. As seen in table 4, PSNR values are robust for cropping, filtering and compression but fails for noise addition. However, SSIM performs fairly better as metric for most of the attacks in comparison to PNSR.

Table 4. Performance of Nested Watermark scheme for robustness

\begin{tabular}{|c|c|c|c|c|c|c|c|c|c|c|c|c|}
\hline \multirow{2}{*}{$\begin{array}{l}\text { Attacks } \backslash \\
\text { Bands }\end{array}$} & \multicolumn{3}{|c|}{ LL } & \multicolumn{3}{|c|}{ LH } & \multicolumn{3}{|c|}{ HL } & \multicolumn{3}{|c|}{ HH } \\
\hline & PSNR & NCC & SSIM & PSNR & NCC & SSIM & PSNR & NCC & SSIM & PSNR & NCC & SSIM \\
\hline $\begin{array}{l}\text { Salt \& Pepper } \\
: 0.01\end{array}$ & 24.1509 & 0.9978 & 0.8215 & 24.4356 & 0.9976 & 0.8315 & 24.0607 & 0.9976 & 0.8214 & 23.5871 & 0.9974 & 0.8024 \\
\hline $\begin{array}{l}\text { Salt \& Pepper } \\
: 0.1\end{array}$ & 24.1488 & 0.9980 & 0.8224 & 14.0401 & 0.9755 & 0.2385 & 14.1088 & 0.9750 & 0.2404 & 14.2384 & 0.9768 & 0.2471 \\
\hline $\begin{array}{l}\text { Gaussian } \\
\text { Noise :0.01 }\end{array}$ & 21.1655 & 0.9169 & 0.3629 & 21.1434 & 0.9157 & 0.3610 & 21.2233 & 0.9166 & 0.3629 & 21.1846 & 0.9177 & 0.3617 \\
\hline $\begin{array}{l}\text { Gaussian } \\
\text { Noise : } 0.1\end{array}$ & 21.1666 & 0.9189 & 0.3610 & 12.1417 & 0.8594 & 0.0976 & 12.1580 & 0.8598 & 0.0965 & 12.1626 & 0.8589 & 0.0974 \\
\hline $\begin{array}{l}\text { Cropping - } \\
1: 128\end{array}$ & 15.7707 & 0.8679 & 0.8074 & 15.7705 & 0.8678 & 0.8070 & 15.7710 & 0.8679 & 0.8073 & 15.7704 & 0.8678 & 0.8069 \\
\hline $\begin{array}{l}\text { Cropping - } \\
150: 256\end{array}$ & 22.1720 & 0.9268 & 0.8836 & 22.1714 & 0.9266 & 0.8832 & 22.1711 & 0.9267 & 0.8832 & 22.1714 & 0.9267 & 0.8830 \\
\hline $\begin{array}{l}\text { Cropping - } \\
200: 256\end{array}$ & 32.1319 & 0.9949 & 0.9823 & 32.1296 & 0.9947 & 0.9818 & 32.1270 & 0.9948 & 0.9819 & 32.1283 & 0.9948 & 0.9817 \\
\hline Blurring & 32.0059 & 0.9965 & 0.9396 & 32.0053 & 0.9965 & 0.9395 & 32.0264 & 0.9964 & 0.9397 & 31.9637 & 0.9964 & 0.9391 \\
\hline
\end{tabular}




\begin{tabular}{|c|c|c|c|c|c|c|c|c|c|c|c|c|}
\hline Sharpening & 16.1239 & 0.9260 & 0.5859 & 16.1104 & 0.9254 & 0.5817 & 16.1081 & 0.9257 & 0.5836 & 16.1243 & 0.9256 & 0.5830 \\
\hline Median filter & 33.6436 & 0.9968 & 0.9520 & 33.6242 & 0.9965 & 0.9511 & 33.6823 & 0.9968 & 0.9518 & 33.6330 & 0.9968 & 0.9515 \\
\hline $\begin{array}{l}\text { Histogram } \\
\text { equalization }\end{array}$ & 9.3403 & 0.9578 & 0.4236 & 9.3424 & 0.9575 & 0.4230 & 9.3385 & 0.9579 & 0.4230 & 9.3476 & 0.9573 & 0.4227 \\
\hline $\begin{array}{l}\text { JPEG } \\
\text { Compression } \\
Q=80 \% \\
\end{array}$ & 39.6831 & 0.9982 & 0.9830 & 39.6725 & 0.9981 & 0.9825 & 39.6790 & 0.9981 & 0.9824 & 32.7716 & 0.9981 & 0.9822 \\
\hline $\begin{array}{l}\text { JPEG } \\
\text { Compression } \\
Q=50 \%\end{array}$ & 36.0422 & 0.9962 & 0.9588 & 36.0712 & 0.9960 & 0.9582 & 36.0522 & 0.9961 & 0.9587 & 36.1438 & 0.9961 & 0.9589 \\
\hline $\begin{array}{l}\text { JPEG } \\
\text { Compression } \\
Q=20 \%\end{array}$ & 32.1198 & 09922 & 0.8991 & 32.1499 & 0.9921 & 0.8986 & 32.1477 & 0.9922 & 0.8984 & 32.1687 & 0.9922 & 0.8981 \\
\hline
\end{tabular}

\section{Conclusion}

With this paper we implemented a nested watermark scheme using a combination of DWT and SVD. The proposed algorithm, we have been able to maximum watermark payload with the dimension of cover image and watermark image being same. Both the watermarks were embedded in LL band against the belief that it will result in the degradation of perceptual quality of the image. However, it was seen with the computation of PSNR, that good imperceptibility of the final cover (watermarked) image is achieved. Proposed scheme performance was also tested for different embedding strength and it showed better for performance for an embedding strength of 0.001. Robustness of the proposed scheme was validated by extracting the watermark artifacts against various attacks. With implementation of the proposed scheme it can be inferred that PSNR can be considered as a good metric for watermarking embedding strength variation. However, PSNR fails to measure image quality for different watermarking artifacts. It is observed that SSIM as metric better for measuring image quality, but fails for histogram equalization. All the objective metrics employed provide good quality assessment of the proposed scheme. Further, we look forward for signature based nested watermarking schemes that provide maximum payload along with authentication. Computational cost and hardware implementation of proposed scheme may be considered for evaluating the performance.

\section{Acknowledgments}

We express our heartfelt gratitude to the college management of BNM Institute of Technology, Benagaluru for enabling us to carry out the research work with all the resources and subsequent publication. Also, we express our sincere gratitude to Visvesvaraya Technological Institute (VTU), Belagavi for providing us the research platform.

\section{REFERENCES}

[1] Begum, Mahbuba; Uddin, Mohammad S. 2020. "Digital Image Watermarking Techniques: A Review" Information 11, no. 2: 110, doi.org/10.3390/info11020110

[2] Akshya Kumar Gupta and Mehul S Raval, "A robust and secure watermarking scheme based on singular values replacement", $\mathrm{Sa}^{-}$dhana- Vol. 37, Part 4, August 2012, pp. 425-440._c Indian Academy of Sciences

[3] Ganic Emir and Ahmet Eskicioglu M 2004, “ Robust DWT-SVD domain image watermarking: Embedding data in all frequencies". Proceedings of the workshop on Multimedia and Security 166-174

[4] Enrico Marini, Florent Autrusseau, Patrick Le Callet, Patrizio Campisi., " Evaluation of standard watermarking techniques". Electronic Imaging, Security, Steganography, and Watermarking of Multimedia Contents, Jan 2007, San jose, United States. pp.6505-24. hal-00250682

[5] Nguyen, Phi Bang, Marie Luong, and Azeddine Beghdadi. "Statistical Analysis of Image Quality Metrics for Watermark Transparency Assessment.” Lecture Notes in Computer Science, 2010, 685-96. doi:10.1007/978-3642-15702-8_63.

[6] K.Silpa and Dr.S.Aruna Mastani, “Comparison Of Image Quality Metrics”, International Journal of Engineering Research \& Technology (IJERT), Vol. 1 Issue 4, June - 2012 ISSN: 2278-0181 